\title{
Perfil de resistencia del VIH-1 a anti-retrovirales en pacientes con fallo virológico. Hospital Roosevelt-Guatemala 2008-2012
}

\author{
Ricardo Mendizábal-Burastero, Amalia C. Girón-Callejas, Jorge A. Rodas-Cruz, \\ Rodolfo Pinzón, M. Lisbeth Romero, E. Marisol Boror y Carlos R. Mejía-Villatoro
}

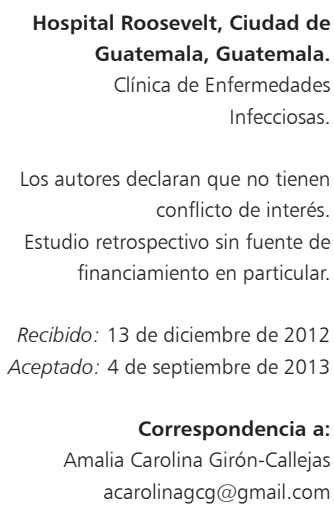

Hospital Roosevelt, Ciudad de Guatemala, Guatemala. Clínica de Enfermedades Infecciosas.

Los autores declaran que no tienen conflicto de interés. Estudio retrospectivo sin fuente de financiamiento en particular.

Recibido: 13 de diciembre de 2012 Aceptado: 4 de septiembre de 2013

Correspondencia a: Amalia Carolina Girón-Callejas acarolinagcg@gmail.com

\section{Patterns of HIV-1 resistance to antiretroviral drugs in patients with virologic failure. Roosevelt Hospital, Guatemala 2008-2012}

Objective: To assess the secondary resistance patterns of HIV-1 to Anti-Retroviral Agents drugs (ART) in patients with virological failure in the main HIV care center in Guatemala. Methods: Using the Stanford HIV Database,HIV pol sequences were analyzed to obtain resistance patterns in patients with first-failure to ART or multiple-failures ( 2 or more regimens failed), from 2008 to 2012. Proportions and odds ratio (OR) with 95\% confidence intervals $(95 \% \mathrm{CI})$ were calculated. Results: $83 \%(43)$ in the first-failure and 75\% (30) in multiple-failures had resistance. The highest frequency (70\%)of resistance was found in the non-nucleoside-inhibitors ART family. 44\% (42) showed resistance to two ART families and 4\% (4) to the three families. First-failure patients had higher risk of nucleoside-inhibitor resistance (OR:3.0, 95\% CI 1.29-6.98) and multidrug resistance (OR:4.94, 95\%CI 1.98-12.32). Most frequent mutations were: M184V, K103N and K65R (71, 50 and 22\%, respectively). 70\% of patients with first-failure were resistant to at least one of the drugs used as second ART in Guatemala (ABC, ddI or AZT). Conclusions: The high level of HIV-1 resistance to ART observed, suggest the need to amend the current second line regimen treatments in Guatemala and the importance of viral genotyping in all patients with first-failure to ART.

Key words: HIV-1, Anti-Retroviral Agents, Drug Resistance, Viral, Mutation, Guatemala.

Palabras clave: VIH-1, anti-retrovirales, resistencia secundaria, mutación, Guatemala.

\section{Abreviaturas de fármacos}

\begin{tabular}{|ll|}
\hline ABC & abacavir \\
ATV/rtv & atazanavir/ritonavir (en baja dosis) \\
AZT & zidovudina \\
ddI & didanosina \\
LPV/rtv & lopinavir/ritonavir (en baja dosis) \\
DRV/rtv & darunavir/ritonavir (en baja dosis) \\
D4T & stavudina \\
ETR & etravirina \\
FTC & emtricitabina \\
IDV/rtv & indinavir/ritonavir (en baja dosis) \\
NFV & nelfinavir \\
NVP & nevirapina \\
SQV & saquinavir \\
SQV/rtv & saquinavir/ritonavir (en baja dosis) \\
TDF & tenofovir \\
3TC & lamividina \\
\hline
\end{tabular}

\section{Introducción}

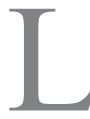

a oferta universal de tratamiento anti-retroviral -TARV- se considera una intervención en salud pública de gran impacto para detener el avance de la epidemia causada por el virus de inmunodeficiencia humana -VIH-. Su importancia y efectividad ha sido ampliamente demostrada, no sólo en el aspecto de mejorar la sobrevida de los pacientes infectados con VIH, sino también como una estrategia de prevención de nuevos casos ${ }^{1}$. Sin embargo, es importante considerar en el ámbito clínico y en salud pública, el riesgo de aparición de cepas de virus resistentes a los fármacos, no sólo por la alta variabilidad genética del virus, sino también como resultado directo de una inadecuada adherencia al TARV, lo cual genera selección natural de cepas resistentes, disminuyendo las opciones terapéuticas y aumentando los costos asociados a tratamientos de rescate. Según la guía de tratamiento de la OMS en el año 2004, se consideraba que la realización de pruebas de resistencia no iba a ser una necesidad a corto o mediano plazo para países en vías de desarrollo, como es el caso de Guatemala, pero en la actualidad, las 
guías internacionales recomiendan en todos los casos de fallo virológico, la realización de prueba de resistencia ${ }^{2,3}$. La guía guatemalteca vigente indica la realización de pruebas de resistencia a TARV solamente en caso de fallo a esquema terapéutico de rescate o de segunda línea ${ }^{4}$.

En Guatemala, a partir del año 2001 se inició la introducción de la TARV de gran actividad -TARGA-; se estimó que para el año 2009 la cobertura de TARV fue de $69 \%$, tratando a 10.362 pacientes $^{5}$. Más de $90 \%$ de los pacientes que reciben TARV se encuentran en esquema de primera línea, siendo la sobrevida a 12 meses de seguimiento de $83 \%$ a nivel nacional ${ }^{5}$. La Clínica de Enfermedades Infecciosas del Hospital Roosevelt constituye el centro de atención integral público con mayor número de pacientes en el país, habiendo iniciado a 4.730 pacientes en TARV entre 2001 y 2012 y manteniendo activos en seguimiento a $70 \%$ de estos; $12 \%$ de los pacientes reciben TARV en esquemas de rescate o de segunda línea y al menos uno de cada 10 de ellos está en fallo virológico. Como esquema de primera línea se utilizan dos inhibidores nucleosídicos de transcriptasa reversa -INTR- (generalmente TDF y FTC, combinación fija) y un inhibidor no nucleosídico -INNTR- (EFV o NVP). Como esquema de rescate, se utilizan dos INTR diferentes (ABC y ddI) y un inhibidor de proteasa -IP- (LPV/rtv) $)^{5-7}$. En la población atendida por esta clínica se ha estimado una prevalencia de resistencia transmitida del VIH a TARV de $8,3 \%$, principalmente asociada a INNTR en $83 \%$.

El TARV para personas con VIH que han fallado al primer esquema o a varios esquemas representa un reto particular en los países con recursos limitados, dado que la mayoría de pacientes han sido tratados inicialmente con medicamentos de baja barrera genética como los INTR e INNTR. El riesgo de este tipo de esquemas, unido a un tiempo prolongado en fallo virológico, aumenta la probabilidad de tener resistencia a más de una familia de anti-retrovirales (ARVs), comprometiendo la selección del esqueleto de INTR del esquema de segunda línea. Esto ya se ha observado en otros países en vías de desarrollo, como lo publicado con respecto a una cohorte tailandesa, donde se observó que las mutaciones de resistencia a INNTR e INTR, luego de fallo con el primer esquema a base de d4T-3TC-NVP en combinación fija, limitaba de manera significativa la terapéutica de segunda línea a $48 \%$ de los pacientes luego del primer fallo ${ }^{9}$. Similares hallazgos fueron observados en una cohorte de Malawi, donde usando el mismo esquema de primera línea, se observó $56 \%$ de mutaciones de resistencia a INNTR e INTR $^{10}$. En la región Centroamericana, solamente en Costa Rica, Honduras y Panamá se ha realizado análisis de resistencia secundaria del VIH en población adulta. En Costa Rica en el año 2003 fueron evaluados 66 casos de fallo virológico, encontrando mutaciones asociadas principalmente a INTR (78\%), en similar proporción para INNTR e IP (50\%), 49\% de los casos evidenciaron resistencia tanto a INTR como a IP y $24 \%$ evidenció resistencia a las tres familias de TARV evaluadas ${ }^{11}$. En el caso de Honduras, entre los años 2004 y 2007 fueron evaluados 97 casos de adultos con fallo virológico, 74\% de ellos evidenciaron fallo virológico, siendo prevalente las mutaciones M184V (62\%) y K103N (30\%) ${ }^{12}$. En el caso de Panamá, fue reportado en el año 2009 un estudio filogenético del VIH incluyendo muestras plasmáticas de $82(61 \%)$ individuos con SIDA y 53 (39\%) pacientes vírgenes a TARV, solamente detectaron mutaciones asociadas a resistencia del VIH en $8(9,7 \%)$ de los pacientes con SIDA $^{13}$.

Este tipo de estudios no se han realizado previamente en Guatemala, siendo el objetivo de este reporte, presentar el análisis de los resultados de laboratorio de pruebas de resistencia del VIH a TARV en pacientes que presentaban fallo virológico durante su atención clínica y seguimiento en la Clínica de Enfermedades Infecciosas, durante el período de 2008 al primer semestre del año 2012, analizando los patrones de resistencia, mutaciones asociadas y evaluando posibles factores de riesgo.

\section{Material y Métodos}

\section{Universo y muestra}

Se realizó el análisis retrospectivo de 96 casos de genotipificación de VIH-1 realizados en muestras sanguíneas de pacientes con fallo virológico que acudieron a la Clínica de Enfermedades Infecciosas, Hospital Roosevelt, entre los años 2008 y primer semestre de 2012. Se incluyeron 56 casos de fallo a primer esquema y 40 casos de fallo múltiple. No se colectaron datos sobre tiempo de encontrarse en TARV. Con respecto a los esquemas, por norma nacional los pacientes de primer esquema tienen como tratamiento dos INTR + un INNTR (generalmente TDF + FTC o AZT + 3TC unido a EFV o NVP); mientras que los pacientes de fallo múltiple son aquellos que han fallado a más de un esquema, y su último esquema es dos INTR + un IP (ABC + ddI o AZT + TDF unido a SQV/ rtv o LPV/rtv).

La selección de pacientes para realización de pruebas de genotipo se realizó en función de los pacientes con fallo virológico que cumplían los requerimientos establecidos en los protocolos internos de la institución, incluyendo evaluación de historia clínica, evolución en el recuento de linfocitos TCD4+, determinaciones de cargas virales de VIH-1, adherencia a TARV, situación social y adicciones, y otros posibles co-factores de fallo terapéutico no relacionados a medicamentos. A partir de octubre de 2009 se realizó la creación de un comité de resistencia de la institución, conformado por un equipo multidisciplinario de profesionales, encargados de realizar la revisión de casos y la selección de pacientes. 


\section{Determinación de fallo virológico}

Para la determinación de fallo virológico, se empleó las guías terapéuticas correspondientes al período analizado. Se definió como fallo virológico al paciente con al menos dos cargas virales mayores a 1.000 copias $\mathrm{ARN} / \mathrm{mL}$, mientras se encontraba en TARV.

Se definió como pacientes en primer fallo, aquellos pacientes que se encontraban en TARV de primera línea, y fallaron a dicho esquema. En el caso de fallo múltiple, el mismo se definió como fallo a más de un esquema anti-retroviral recibido.

\section{Realización de carga viral y prueba de genotipificación de VIH-1}

La determinación de carga viral para el seguimiento virológico de los pacientes se realizó por medio de la metodología COBAS Amplicor (Roche Diagnostics, CA) durante el año 2007; a partir del año 2008 se realizó por medio de la metodología COBAS Ampliprep/ COBAS Taqman (Roche Diagnostics, CA). En ambos casos, se trabajó de acuerdo a las especificaciones del fabricante.

En el caso de la determinación de genotipo para VIH-1, fueron realizados por diversas instituciones y con diferentes metodologías, se incluyeron resultados obtenidos por medio de la técnica Virco (Virco Laboratories), Viroseq HIV-1 Genotyping (Abbott, Wiesbaden, Germany), Trugene HIV-1 Genotyping (Siemens Healthcare Diagnostics, Eschborn, Germany); a partir del año 2011, la mayoría de los ensayos se realizaron en la Clínica de Enfermedades Infecciosas del Hospital Roosevelt, mediante la metodología Trugene HIV-1 Genotyping.

\section{Análisis de perfil de resistencia}

Se realizó el análisis de perfil de resistencia basado en los resultados de genotipo, por medio de la base de datos Stanford HIV database ${ }^{14,15}$. Se utilizó tanto la puntuación como la interpretación de la misma. Posible/bajo nivel de resistencia corresponde a Stanford score (SS) de 10-29, nivel intermedio de resistencia a SS de 30-59 y alto nivel de resistencia a $S S \geq 60$. Los datos fueron tabulados y analizados en una base de datos de diseño propio elaborada en EpiInfo 3.5.3.

\section{Análisis de datos}

Se realizó estadística descriptiva para la caracterización de la muestra. Se determinó la mediana y cuartiles de edad y carga viral. Se calculó la proporción de resistencia a ARVs, agrupada por familia y medicamento.

Se realizó análisis de riesgo para variables dicotómicas, prueba de $\chi^{2}$ para variables categóricas y prueba de $\mathrm{Z}$ para comparación de medias. Se trabajó con $5 \%$ de significancia.

\section{Resultados}

De los 96 casos analizados, 52\% (n: 50) de los casos fueron de género masculino, siendo la mediana de edad 32 años $\left(Q_{1} 27,5-Q_{3} 38,0\right)$. En la Figura 1 se puede observar una distribución muy similar por género en los dos tipos de fallo. Se observa además que la mediana de edad en ambos grupos es muy similar, pero con un rango intercuartílico menor en el caso de los pacientes en fallo múltiple. Se realizó prueba de comparación de medias entre tipo de fallo, donde no se encontró diferencia por edad (p: $0,912)$ pero sí se observó diferencia significativa para log de carga viral (p: 0,033). El 76\% (n: 73) de los casos evidenció resistencia del VIH a los ARVs. No existió diferencias por tipo de fallo y género (p: 0,318 y p: 0,160, respectivamente). De igual manera, se realizó prueba de asociación para relación entre presencia de mutaciones por familia de ARV y carga viral, encontrando una relación significativa entre carga viral $<50.000$ copias $/ \mathrm{mL}$ y mutaciones a INTR + INNTR (p: 0,003).

Con respecto a la prevalencia de resistencia por familia de ARVs, la mayor frecuencia de resistencia se observa en los INNTR, seguida por los INTR, en ambos grupos de pacientes. Se observa una mayor frecuencia de resistencia a IP en los pacientes con fallo múltiple. Los pacientes con primer fallo presentaron tres veces más riesgo para resistencia INTR que los pacientes con fallo múltiple (OR: 3,00 IC 95\% 1,29-6,98). De manera similar, en el caso de multi-resistencia a INTR +INNTR los pacientes de primer fallo presentan cinco veces más riesgo que los pacientes de fallo múltiple (OR: 4,4; IC 95\% 1,98-12,32).

Para el análisis de mutaciones y perfil de resistencia, se consideró solamente a los 73 pacientes con evidencia de resistencia. Como se observa en la Figura 1, la mutación más frecuentemente detectada en los INTR fue M184V, en $71 \%$ de los pacientes, $(90 \%$ en pacientes de primera línea), seguida de la K65R detectada en 22\% de los pacientes (33\% en pacientes de primera línea). El 69\% de los pacientes con K65R tenían al menos una de las mutaciones Y181C o G190A/S.

En el caso de los INNTR, se observó una frecuencia alta de $\mathrm{K} 103 \mathrm{~N}$, en $50 \%$ de los pacientes $(51 \%$ en los pacientes de primera línea), seguido por Y181C y G190A/S. Con respecto a las mutaciones asociadas a análogos de timidina (MAATs), se encontraron cuatro pacientes con una MAAT, tres pacientes con dos MAATs (K70R+D67N en dos casos y M41L+T215Y) y dos pacientes con tres MAATs (M41L+D67N+T215Y y M41L+L210W+T215Y). En todos los casos de múltiples MAATs, eran pacientes con fallo a múltiples esquemas. Sólo se observó un paciente con mutación Q151M, y no se observó casos de inserción 69.

En el caso de los pacientes cuyo genotipo de VIH-1 evidenció mutaciones en el gen de la proteasa, se observó 


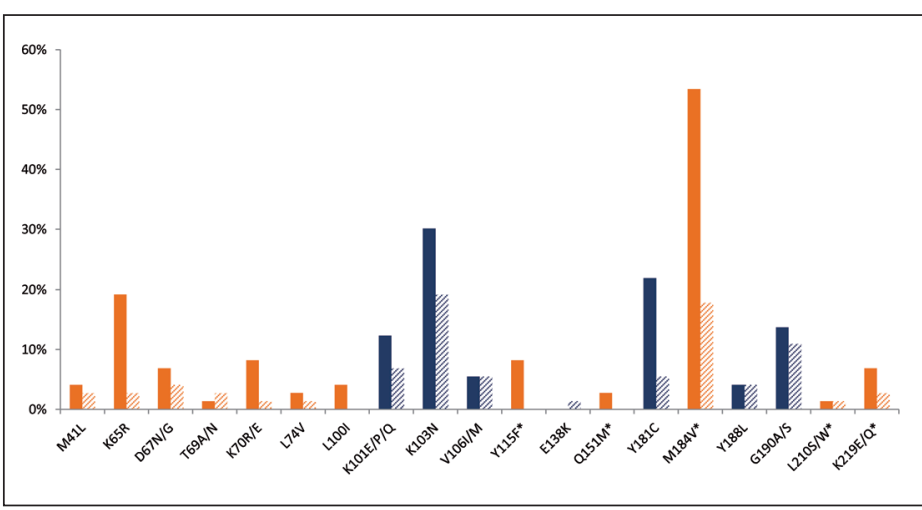

Figura 1. Proporción de mutaciones de resistencia en el gen codificante de la transcriptasa reversa del VIH-1 (n: 73), Hospital Roosevelt-Guatemala, 2008-2012. Barras sólidas: Primer fallo; Barras con líneas en diagonal: Fallo múltiple; Color naranja: Mutaciones para INTR; Color azul: Mutaciones para INNTR.

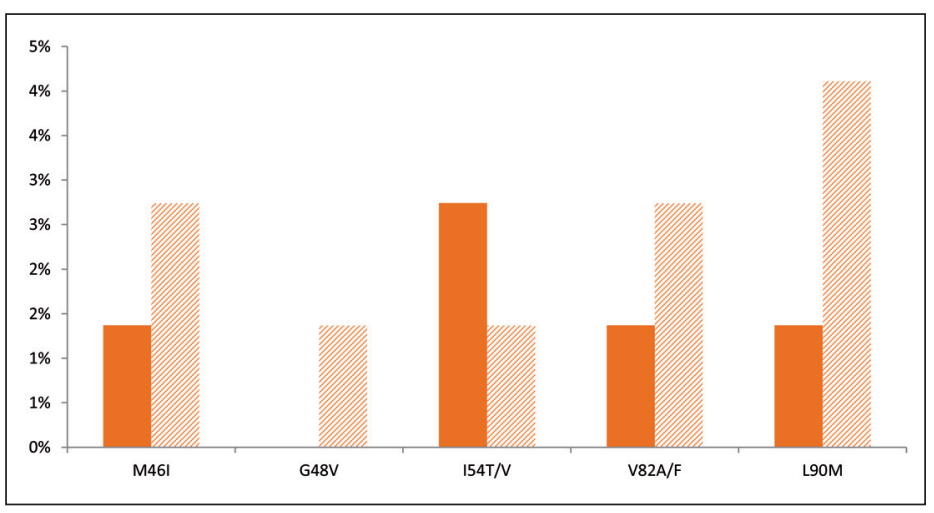

Figura 2. Proporción de mutaciones de resistencia en el gen codificante de la proteasa del VIH-1 (n: 73), Hospital Roosevelt-Guatemala, 2008-2012. Barras sólidas: Primer fallo; Barras con líneas en diagonal: Fallo múltiple.

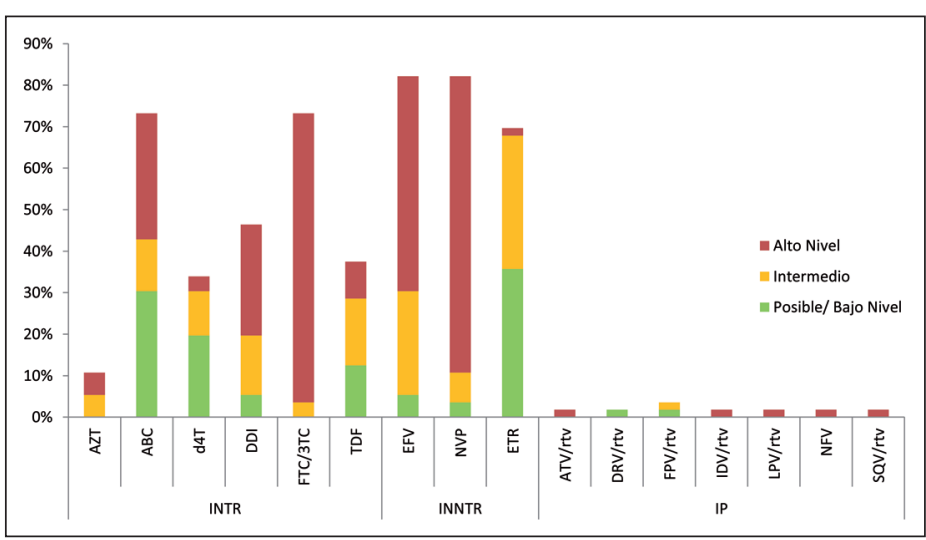

Figura 3. Perfil de resistencia del $\mathrm{VIH}-1$ en pacientes con fallo a primer esquema (n: 56), Hospital Roosevelt-Guatemala, 2008-2012. Posible/bajo nivel de resistencia corresponde a Stanford score (SS) de 10-29, nivel intermedio de resistencia a SS de 30-59 y alto nivel de resistencia a $S S \geq 60$. Nota: AZT: zidovudina, ABC: abacavir, D4T: stavudina, DDI: didanosina, FTC: emtricitabina, 3TC: lamivudina, TDF: tenofovir, EFV: efavirenz, NVP: nevirapina, ETR: etravirina, ATV/r: atazanavir con ritonavir, DRV/r: darunavir con ritonavir, FPV/r: fosamprenavir con ritonavir, IDV/r: indinavir con ritonavir, LPV/r: lopinavir con ritonavir, NFV: nelfinavir, SQV/r: saquinavir con ritonavir.

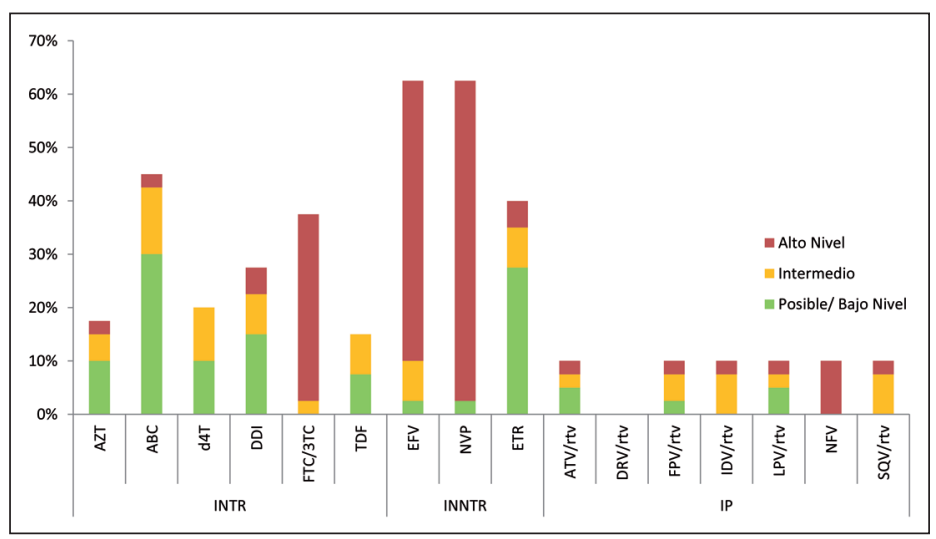

Figura 4. Perfil de resistencia del VIH-1 en pacientes con fallo a múltiples esquemas (n: 40), Hospital Roosevelt-Guatemala, 2008-2012. Posible/bajo nivel de resistencia corresponde a Stanford score (SS) de 10-29, nivel intermedio de resistencia a SS de 30-59 y alto nivel de resistencia a $S S \geq 60$. Nota: AZT: zidovudina, $A B C$ : abacavir, D4T: stavudina, DDI: didanosina, FTC: emtricitabina, 3TC: lamivudina, TDF: tenofovir, EFV: efavirenz, NVP: nevirapina, ETR: etravirina, ATV/r: atazanavir con ritonavir, DRV/r: darunavir con ritonavir, FPV/r: fosamprenavir con ritonavir, IDV/r: indinavir con ritonavir, LPV/r: lopinavir con ritonavir, NFV: nelfinavir, SQV/r: saquinavir con ritonavir. una frecuencia baja de mutaciones importantes de resistencia (Figura 2). La mayor proporción se observó en polimorfismos asociados a bajo nivel de resistencia, como en el caso de L10I/V y A71T/V, alrededor de $25 \%$ cada una. Se observa una proporción más alta de mutaciones de IPs mayores en pacientes de fallo múltiple, principalmente en L90M y G48V.

Con respecto al perfil de resistencia en pacientes con fallo a primer esquema (Figura 3), la mayor resistencia se observó en la familia de INNTR, con resistencia cerca de $80 \%$ en EFV y NVP. En el caso de los INTR, se observa una proporción alta en FTC/3TC y en ABC.
En este grupo de pacientes, $40 \%$ presentó resistencia a ABC y ddI, y 70\% de los pacientes presentó resistencia a al menos uno de los medicamentos utilizado de acuerdo al Protocolo Nacional como segunda línea o de rescate (ABC, ddI o AZT).

En la Figura 4, se presenta el perfil de resistencia de los pacientes en fallo a múltiples esquemas. Se observó una mayor proporción de resistencia en los IP que en los pacientes con fallo a primer esquema, encontrando una mayor frecuencia en NFV. En el caso de los INTR, los ARVs más afectados fueron FTC/3TC con un alto nivel de resistencia y $\mathrm{ABC}$ con intermedio y bajo nivel 
de resistencia. Con respecto a los INNTR, predominó la resistencia a EFV y NVP.

\section{Discusión}

La resistencia a los ARVs constituye un grave problema, no sólo clínico, debido a sus implicaciones sobre las opciones terapéuticas de los pacientes seropositivos para VIH, sino también un problema de salud pública, que determina la manera de diseñar y seleccionar medicamentos para ser incluidos en los protocolos de tratamiento de los países. En este reporte se observa la alta frecuencia de resistencia a INTR e INNTR en pacientes de primer fallo, causando en estos pacientes una notable disminución en sus opciones terapéuticas.

En el aspecto de los pacientes de primer fallo, se observa de manera esperada, un nivel alto de resistencia a los INNTR, principalmente a EFV y NVP. Esto es debido a la baja barrera genética de estos medicamentos, como ha sido demostrado ampliamente en otros estudios. La presencia de mutaciones como K103N e Y181C se encuentra altamente correlacionada con la utilización previa de NVP y EFV ${ }^{16-18}$. También es importante considerar la prevalencia de resistencia cruzada a ETR, lo que limita su utilización como posible alternativa terapéutica, en al menos dos de cada cinco pacientes analizados. El nivel de resistencia encontrado en los INNTR, cercano a $80 \%$, demuestra que debe ser oportuno el cambio de un INNTR por un IP como tratamiento de rescate según está establecido en el Manual de Tratamiento Anti-retroviral y de Infecciones Oportunistas en Guatemala ${ }^{4}$. La adherencia es un aspecto relacionado directamente con el fallo virológico y la resistencia a este tipo de medicamentos, pero la acumulación de varias mutaciones en un mismo paciente hace pensar en un tiempo de fallo prolongado. Debido al enfoque de este reporte, no se correlacionó con aspectos de adherencia, pero debe ser un factor importante a considerar para nuevas investigaciones.

La resistencia a los INTR en los pacientes con fallo virológico al primer esquema terapéutico es el problema más importante en este grupo analizado, ya que no sólo compromete las opciones terapéuticas futuras en el individuo; también evidencia la necesidad de modificar el esquema de rescate en el protocolo nacional y de incluir de manera obligatoria, la prueba de genotipo para pacientes con fallo virológico al primer esquema terapéutico, tal y como fue evidenciado en el estudio controlado y de asignación aleatoria de Viradapt que reportó en el año 2000 el beneficio a largo plazo de la administración de TARV basada en un genotipo viral ${ }^{19}$.

En el caso de las mutaciones a los INTR, es importante destacar la alta frecuencia de M184V y K65R; este hallazgo difiere de lo encontrado en el análisis de resistencia primaria realizado en esta misma clínica, en el cual no se detectó ni la M184V ni la K65R, atribuible a que ambas mutaciones causan una disminución en la capacidad replicativa del virus ${ }^{8}$. Ahora bien, la alta proporción de estas mutaciones en la resistencia secundaria del VIH, seleccionadas por el tratamiento de primera línea (TDF + FTC o TDF + 3TC), limita la disponibilidad de opciones como terapia de rescate, por ejemplo disminuyen la susceptibilidad a ABC y ddI, fármacos utilizados como parte del esquema de rescate en Guatemala ${ }^{20-22}$. Esta disminución en la susceptibilidad está relacionada con la selección cruzada de mutaciones, la mutación M184V le confiere a $\mathrm{ABC}$ un bajo nivel de resistencia y K65R confiere a $\mathrm{ABC}$ un nivel intermedio de resistencia; la sumatoria de ambas mutaciones se traduce en un alto nivel de resistencia a $\mathrm{ABC}^{23}$. Como era de esperarse, según publicaciones a nivel mundial, y de manera similar con lo observado en otros países latinoamericanos, como lo reportado por Taylor-Castillo en Costa Rica, por Murillo en Honduras y por Gómez en Colombia, la M184V fue de las mutaciones más comúnmente observadas ${ }^{11,12,24}$. En el caso de la mutación K65R, ésta se caracteriza por ser rara vez seleccionada, con una incidencia global de 2 a $5 \%$ en los pacientes con genotipificación, a pesar del uso cada vez mayor de TDF y ABC desde el año 200121,25; sin embargo, en este análisis realizado en Guatemala, la frecuencia de detección de K65R (22\%), fue mucho mayor al 2-5\% esperado. Esta situación podría atribuirse a que en los países con recursos limitados se han utilizado medicamentos como NVP, d4T, 3TC, AZT o ddI, que se han asociado a efectos tóxicos, afectando la adherencia al tratamiento o que suelen ser sub-óptimos y que no necesariamente logran una adecuada supresión viral, situación que conduce a una más rápida aparición de $\mathrm{K} 65 \mathrm{R}^{21}$. Tal hecho fue reportado por Gallant y cols., quienes detectaron $21 \%$ de K65R en la combinación de TDF + 3TC que no permitió obtener una adecuada supresión viral ${ }^{26}$. Aunado a esto, se ha evidenciado una fuerte asociación para la selección de K65R con el uso de NVP y sus mutaciones de resistencia Y181C/G y G190A/S $\mathrm{S}^{27-30}$. Interesantemente, en el caso de Guatemala, todos los pacientes con K65R tenían antecedente de uso, además de TDF, al menos uno de los siguientes: NVP, d4T, AZT o ddI; sumado a esto, más de dos tercios tenían al menos una de estas mutaciones Y181C/G o G190A/S.

Los hallazgos del perfil de resistencia encontrados para INRT e INNRT hacen evidente la necesidad en Guatemala de realizar una prueba de resistencia luego del fallo a primer esquema. Según lo observado en otros estudios, la inclusión de la prueba de genotipo para optimizar el tratamiento, idealmente acompañado de un comité de expertos para el análisis del mismo, mejora notablemente el éxito virológico del nuevo tratamiento administrado ${ }^{31,32}$. En nuestra institución, desde el año 2009 se cuenta con di- 
cho comité, para la evaluación de candidatos para prueba de genotipificación y las posteriores recomendaciones de tratamiento, lo cual permite un mejor aprovechamiento de los recursos, pero es necesario que el Ministerio de Salud Pública provea nuevas opciones terapéuticas y adquiera los insumos necesarios para aumentar la disponibilidad de pruebas de genotipificación.

En pacientes con resistencia a más de una familia de ARV, las expectativas de tratamiento se reducen notablemente. Según los protocolos terapéuticos vigentes en Guatemala, se propone el TARV basado solamente en tres familias de ARV (INTR, INNTR e IP); en este estudio se evidenció que $60 \%$ de los pacientes de primera línea presentaban resistencia a INTR e INNTR conjunta; unido a esto, $70 \%$ de los pacientes presentó resistencia a al menos un medicamento INTR de rescate, indicando la necesidad de contar con nuevas familias de tratamientos, como podría ser el caso de inhibidores de fusión o de la integrasa. En una cohorte de pacientes italianos, Zacarelli comprobó la disminución en la supervivencia en los pacientes con múltiple resistencia a varias familias ${ }^{33}$. Además, en las cohortes de TORO 1 y TORO 2, se observó un mejor pronóstico en pacientes que contaban con tres ARVs activos ${ }^{34,35}$. En el caso de Guatemala, este análisis evidenció menor nivel de multi-resistencia que el detectado en países de la región; en Guatemala la proporción de resistencia a tres familias de ARVs fue de $4,2 \%$, mientras que en Costa Rica y Honduras fue de 24 y $27 \%$, respectivamente ${ }^{11,12}$. Sin embargo, es interesante observar que en el grupo de pacientes de Guatemala, se evidenció un mayor riesgo de tener resistencia a dos familias en pacientes con fallo a primera línea que en pacientes en fallo múltiple. Esto pareciera diferir con lo observado con otros autores, como Murillo en Honduras y Napravnik en la cohorte de la Universidad de Carolina del Norte, donde se encontró una correlación entre exposición previa extensiva con la presencia de multi-resistencia ${ }^{12,36}$. En nuestro grupo de pacientes evaluados, la posible explicación puede ser, en el caso de fallo a primer esquema, que debido a la baja barrera genética de los INNTR y el tiempo prolongado en el que el paciente se encuentra en fallo virológico, se acumularon mutaciones asociadas a los INTR. En el caso de los pacientes de fallo múltiple, donde se esperaría una mayor prevalencia de multi-resistencia, la explicación podría ser la falta de presión farmacológica por inadecuada adherencia, con un consiguiente aumento de cepas salvajes; sin embargo, esta es una limitante de este reporte, la información para responder a estas interrogantes no fue recolectada.

Otro aspecto a considerar es que se observaron cargas virales menores en los pacientes con resistencia a dos familias (INTR y INNTR) en comparación con los que no presentaban resistencia, hallazgos congruentes con la dinámica viral, y la menor capacidad replicativa que tiene los virus con mutaciones de resistencia en comparación con el virus salvaje ${ }^{37}$.

Debe ser considerado en futuros estudios de este grupo de pacientes el tiempo que permanecen en fallo virológico. La frecuencia del monitoreo de carga viral y recuento de linfocitos T CD4+ debe realizarse cada seis meses, de acuerdo a las guías nacionales, pero no existe un protocolo de respuesta rápida y seguimiento de posibles fallos virológicos. Esto puede incidir directamente en la acumulación de mutaciones, disminuyendo las opciones terapéuticas. Actualmente, en nuestra institución se trabaja para implementar un protocolo de detección temprana de fallo virológico, que busca reducir a dos meses el tiempo para confirmación de fallo y extracción de muestra para hacer el ensayo de genotipificación.

Es necesario considerar los aspectos que puedan fortalecer un mejor apego al tratamiento por parte del paciente, incluyendo estrategias con un enfoque comunitario y familiar, con el objetivo de disminuir la cantidad de pacientes en fallo virológico y, por consiguiente la transmisión de estos virus resistentes ${ }^{6}$. En ese sentido, la administración de TARV como estrategia de prevención puede ver limitada su nivel de acción, con el creciente aumento de cepas resistentes, fallando así al objetivo de disminuir la carga viral comunitaria ${ }^{38}$.

\section{Conclusiones}

El alto nivel de resistencia del VIH-1 a los ARVs, observada en pacientes después de primer fallo y fallo múltiple, sugiere la necesidad de modificar el actual esquema terapéutico de rescate en Guatemala y la importancia de realizar el estudio genotípico en todos los pacientes luego del fallo al primer esquema terapéutico, a fin de realizar el cambio de tratamiento basado en evidencia científica.

Agradecimientos: Los autores agradecen la valiosa colaboración de las siguientes personas: Elizabeth Yax, Andrés Moreira y Grethel Lemus como apoyo para el análisis de expedientes y digitación de datos; Verónica García y Miriam Hernández por la búsqueda de expedientes.

\section{Resumen}

Objetivo: Evaluar el perfil de resistencia secundaria del VIH-1 a anti-retrovirales (ARV) en pacientes con fallo virológico en la clínica de atención integral más grande de Guatemala. Métodos: Uso de Stanford HIV Database para análisis de secuencias pol para perfiles de resistencia de VIH en pacientes con fallo virológico al primer esquema ARV o fallo múltiple (dos o más esquemas ARV fallidos), entre los años 2008 y 2012. Determinación de proporciones y análisis de riesgo. Resultados: Evidencia 
de resistencia de $83 \%$ (n: 43) en primer fallo y 75\% (n: 30) en fallo múltiple. La mayor frecuencia de resistencia se presentó en los inhibidores-no-nucleosídicos (70\%). Cuarenta y cuatro por ciento (n: 42) evidenció resistencia a dos familias de ARV y 4\% (n: 4) a las tres familias. Pacientes con primer fallo tuvieron más riesgo de resistencia a inhibidores-nucleosídicos (OR: 3,0; IC 95\% 1,29-6,98) y más riesgo de multi-resistencia (OR: 4,94; IC 95\% 1,98-12,32). Mutaciones más frecuentes fueron: M184V,
K103N y K65R (71, 50 y 22\%, respectivamente). Setenta por ciento de los pacientes con primer fallo presentaron resistencia a al menos uno de los medicamentos utilizado como segunda línea en Guatemala (ABC/ddI/AZT). Conclusiones: El alto nivel de resistencia del VIH-1 a los ARV observada, sugiere la necesidad de modificar el actual esquema terapéutico de rescate en Guatemala y la importancia de realizar genotipificación viral en todos los pacientes con fallo al primer esquema.

\section{Referencias bibliográficas}

1.- $\quad$ Blower S, Ma L, Farmer P, Koenig S Predicting the impact of antiretrovirals in resource-poor settings: preventing HIV infections whilst controlling drug resistance. Curr Drug Targets Infect Disord 2003; 3 (4): 345-53.

2.- Wainberg M A, Friedland G. Public health implications of antiretroviral therapy and HIV drug resistance. JAMA 1998; 279 (24): 1977-83.

3.- World Health Organization. Scaling up antiretroviral therapy in resource-limited settings: treatment guidelines for a public health approach[internet]. World Health Organization; 2004 [Consultado 20 julio 2012]. Disponible en: http://www.who.int/3by5/ publications/documents/arv_guidelines/en/ index.html.

4.- Programa Nacional de Prevención y Control de las ITS VyS. Manual de Tratamiento Antirretroviral y de Infecciones Oportunistas en Guatemala[internet]. Guatemala: Ministerio de Salud Pública y Asistencia Social; 2012 [Consultado 20 julio 2012]. Disponible en: http://www.aidstar-one.com/sites/default/files/ Guatemala\%202012.pdf

5.- Programa Nacional de Prevención y Control de ITS VyS. Reporte UNGASS Guatemala 2010 [internet]. Guatemala: ONUSIDA2010 [Consultado 24 julio 2012]. Disponible en: http://data.unaids.org/Pub/report/2010/ guatemala_2010_country_progress_report es.pdf

6.- Organización Panamericana de la Salud. Tratamiento antirretroviral bajo la lupa: un análisis de salud pública en Latinoamérica y el Caribe[internet]. Mónica Alonso González ed. Washington, D.C.: Organización Panamericana de la Salud; 2012 [Consultado 24 julio 2012]. Disponible en: http://www.paho.org/hq/ index.php?option $=$ com_docman\&task $=$ doc view\&gid $=21388 \&$ Itemid $=$

7.- $\quad$ Mejía C, Romero J, Rodríguez D, editors. HAART under Restricted Circumstances in the Capital of Guatemala[internet]. The 3rd IAS Conference on HIV Pathogenesis and Treatment; 2005 [Consultado 24 julio
2012]. Disponible en: http://iset.aids2010.org/ Abstracts/A2176734.aspx.

8.- Ávila-Ríos S, Mejía-Villatoro CR, García-Morales C, Soto-Nava M, Escobar I, Mendizabal R, et al. Prevalence and patterns of HIV transmitted drug resistance in Guatemala. Rev Panam Salud Pública 2011; 30 (6): 641-8.

9.- Sungkanuparph S, Manosuth W, Kiertiburanakul S, Piyavong B, Chumpathat N, Chantratita W. Options for a second-line antiretroviral regimen for HIV type 1-infected patients whose initial regimen of a fixed-dose combination of stavudine, lamivudine, and nevirapine fails. Clin Infect Dis 2007; 44 (3): 447-52.

10.- Hosseinipour M C, van Oosterhout J J, Weigel R, Phiri S, Kamwendo D, Parkin N, et al. The public health approach to identify antiretroviral therapy failure: high-level nucleoside reverse transcriptase inhibitor resistance among Malawians failing first-line antiretroviral therapy. AIDS 2009; 23 (9): 1127-34.

11.- Taylor-Castillo L, Herrera-Martínez G, León-Bratti MP, Boza R, León-Rodríguez B, Luftig RB, et al. Study of antiretroviral mutants in HIV patients with treatment failures and the effect of risk factors in the virological failures. Rev Inst Med Trop Sao Paulo 2005 Nov-Dec; 47 (6): 327-31.

12.- Murillo W, De Rivera I, Parham L, Jovel E, Palou E, Karlsson A, et al. Prevalence of drug resistance and importance of viral load measurements in Honduran HIVinfected patients failing antiretroviral treatment HIV Medicine 2010; 11 (2): 95-103.

13.- Ahumada-Ruiz S, Flores-Figueroa D, Toala-González I, Thomson M M. Analysis of HIV-1 pol sequences from Panama: identification of phylogenetic clusters within subtype B and detection of antiretroviral drug resistance mutations. Infect Genet Evol 2009 Sep; 9 (5): 933-40.

14.- Liu T F, Shafer R W. Web resources for HIV type 1 genotypic-resistance test interpretation. Clin Infect Dis 2006; 42 (11): 1608-18.

15.- Rhee S-Y, Gonzáles M J, Kantor R, Betts B J, Ravela J, Shafer R W. Human immunodeficiency virus reverse transcriptase and protease sequence database. Nucleic Acids Res 2003; 31 (1): 298-303.

16.- Deeks S G. International perspectives on antiretroviral resistance. Nonnucleoside reverse transcriptase inhibitor resistance. J Acquir Immune Defic Syndr 2001 Mar 1; 26 Suppl 1: S25-S33.

17.- Maggiolo F, Airoldi M, Kleinloog H D, Callegaro A, Ravasio V, Arici C, et al. Effect of adherence to HAART on virologic outcome and on the selection of resistance-conferring mutations in NNRTI-or PI-treated patients. HIV Clin Trials 2007; 8 (5): 282-92.

18.- Parienti J-J, Massari V, Descamps D, Vabret A, Bouvet E, Larouzé B, et al. Predictors of virologic failure and resistance in HIV-infected patients treated with nevirapine-or efavirenzbased antiretroviral therapy. Clin Infect Dis 2004; 38 (9): 1311-6.

19.- Clevenbergh P, Durant J, Halfon P, del Giudice P, Mondain V, Montagne N, et al. Persisting long-term benefit of genotype-guided treatment for HIV-infected patients failing HAART. The Viradapt Study: week 48 followup. Antivir Ther 2000 Mar; 5 (1): 65-70.

20.- Soriano V, Mendoza Cd. Genetic mechanisms of resistance to NRTI and NNRTI. HIV Clin Trials 2002; 3 (3): 237-48.

21.- Brenner BG, Coutsinos D. The K65R mutation in HIV-1 reverse transcriptase: genetic barriers, resistance profile and clinical implications. HIV Therapy 2009; 3 (6): 583-94.

22.- Nicastri E, Sarmati L, d'Ettorre G, Palmisano L, Parisi S G, Uccella I, et al. Replication capacity, biological phenotype, and drug resistance of HIV strains isolated from patients failing antiretroviral therapy. J Med Virol 2003; 69 (1): 1-6.

23.- Moyle G. Resistance and cross-resistance to abacavir. HIV Medicine 2001; 2 (3): 154-62.

24.- Gómez SM, Olaya P, Díaz FJ. Resistencia a los medicamentos antirretrovirales en pacientes que reciben tratamiento para VIH-sida en Colombia. Infectio 2011; 14 (4): 248-57.

25.- McColl D J, Chappey C, Parkin N T, Miller M D. Prevalence, genotypic associations and phenotypic characterization of K65R, L74V and other HIV-1 RT resistance mutations in a 
commercial database. Antivir Ther 2008; 13 (2) 189-97.

26.- Gallant J E, Staszewski S, Pozniak A L, De Jesus E, Suleiman J M, Miller M D, et al. Efficacy and safety of tenofovir DF vs stavudine in combination therapy in antiretroviral-naive patients: a 3-year randomized trial. JAMA 2004; 292 (2): 191-201.

27.- de Mendoza C, Jiménez-Nacher I, Garrido C, Barreiro P, Poveda E, Corral A, et al. Changing patterns in HIV reverse transcriptase resistance mutations after availability of tenofovir. Clin Infect Dis 2008; 46 (11): 1782-5.

28.- von Wyl V, Yerly S, Boni J, Burgisser P, Klimkait T, Battegay M, et al. Factors associated with the emergence of $\mathrm{K} 65 \mathrm{R}$ in patients with HIV-1 infection treated with combination antiretroviral therapy containing tenofovir. Clin Infect Dis 2008; 46 (8): 1299-309.

29.- Rey D, Hoen B, Chavanet P, Schmitt MP, Hoizey G, Meyer P, et al. High rate of early virological failure with the once-daily tenofovir/lamivudine/nevirapine combination in naive HIV-1-infected patients. J Antimicrob Chemother 2009; 63 (2): 380-8.

30.- Clotet B. Once-daily dosing of nevirapine in HAART. J Antimicrob Chemother 2008 Jan; 61 (1): $13-6$

31.- Tural C, Ruiz L, Holtzer C, Schapiro J, Viciana P, González J, et al. Clinical utility of HIV-1 genotyping and expert advice: the Havana trial. AIDS 2002; 16 (2): 209-18.

32.- Badri S M, Adeyemi O M, Max B E, Zagorski B M, Barker D E. How does expert advice impact genotypic resistance testing in clinical practice? Clin Infect Dis 2003; 37 (5): 708-13.

33.- Zaccarelli M, Tozzi V, Lorenzini P, Trotta MP, Forbici F, Visco-Comandini U, et al. Multiple drug class-wide resistance associated with poorer survival after treatment failure in a cohort of HIV-infected patients. AIDS 2005; 19 (10): 1081-9.

34.- Lalezari J P, Henry K, O’Hearn M, Montaner J S, Piliero P J, Trottier B, et al. Enfuvirtide, an HIV-1 fusion inhibitor, for drug-resistant HIV infection in North and South America. N Engl J Med 2003; 348 (22): 2175-85.

35.- Lazzarin A, Clotet B, Cooper D, Reynes J, Arastéh K, Nelson M, et al. Efficacy of enfuvirtide in patients infected with drugresistant HIV-1 in Europe and Australia. N Engl J Med 2003; 348 (22): 2186-95.

36.- Napravnik S, Keys J R, Quinlivan E B, Wohl D A, Mikeal O V, Eron Jr J J. Triple-class antiretroviral drug resistance: risk and predictors among HIV-1-infected patients. AIDS 2007; 21 (7): 825-34.

37.- Lucas G M, Gallant J E, Moore R D. Relationship between drug resistance and HIV-1 disease progression or death in patients undergoing resistance testing. AIDS 2004; 18 (11): 1539-48.

38.- Das M, Chu P L, Santos G-M, Scheer S, Vittinghoff E, McFarland W, et al. Decreases in community viral load are accompanied by reductions in new HIV infections in San Francisco. PloS one 2010; 5 (6): e11068. 